\title{
The Spatio-temporal Disparities in Healthy Food Accessibility: A Case Study of Shanghai, China
}

\author{
Mengqi Zhong, Tongji University; China \\ Yifan Yu, Tongji University; China
}

\begin{abstract}
The supply of healthy food is distributed unequally in city. The accessibility of healthy foods is affected by both locations and traffic conditions. This paper examines spatio-temporal disparities in healthy food accessibility in Shanghai communities. Firstly, we choose all communities in Shanghai and use python as a crawling tool to collect healthy food store POI (e.g. agricultural markets, vegetable markets, fruit markets, aquatic seafood markets, supermarkets and comprehensive markets) from Gaode Map and get 23,436 points to calculate the amount and density of healthy food store in various communities. Secondly, after comparing Baidu Map and Gaode Map, leading platforms of Web GIS services in China, we choose Baidu Map to collect data to study the spatio-temporal difference in accessibility by using network analysis and developing a crawling tool to collect different travel time (e.g. walking and public transportation) for each community to the closest healthy food store at each time of day (0:00-24:00). Thirdly, we set up a variable to see at what time are people in the communities able to reach their nearest healthy food store in 15 minutes and the ratio of the above-mentioned time to the whole day is calculated so that we can evaluate the temporal disparities of healthy food accessibility. Additionally, we use global and local spatial autocorrelation to analyze the spatial patterns of the temporal disparities of healthy food accessibility, based on the Moran's index and the local indicator spatial association (LISA) index. Finally, on the basis of the research above, the food desert map is drawn. The results of this analysis identify the communities in Shanghai with the greatest need for improved access to healthy food stores and the variance of accessibility affected by the traffic in different times will be taken into account. Ultimately, this study explores a more complete and realistic condition of healthy food accessibility in Shanghai and the corresponding improvement strategy is proposed.
\end{abstract}

\section{Keywords}

healthy food accessibility, spatio-temporal disparities, food deserts, spatial autocorrelation 


\section{Introduction}

\subsection{Background}

Obesity rates have risen sharply in recent decades, and according to the World Health Organization, more than one in ten people in the world are now classified as obese (WHO,2013). The rise in obesity rates is blamed on increased consumption of sweet and energy-dense foods (WHO,2002). A series of studies have shown that diet can affect health and unhealthy dietary intake is associated with a higher incidence of multiple adverse health outcomes (World Cancer Research Fund/American Institute for Cancer Research, 2007), including many major causes of death such as heart disease and various types of cancer (Jamie Pearce et al.,2009). High density of fast-food restaurants and corner stores that offer prepared foods is always related to unhealthy dietary intake, while easier access to healthy food stores, like fruits and vegetables stores, should improve the residents' diet nutrition and subsequently enhance their well-being and health(key,2011; Kent and Thompson, 2014, Story et al., 2008, Taylor, 2015).It's important to choose healthy food for health promotion, while there are many factors that influence people's choices. Factors affecting dietary intake have been well-explained at the socio-economic level including educational level, employment status, income and cultural differences (Dowler, 2001). As research progresses, scholars are paying more and more attention to the supply of healthy foods in the community. People tend to make food choices based on the food access in their immediate neighborhood (Furey et al., 2001), which suggests that factors in the built environment play a key role in people's diets (Morland et al., 2002b; Rose and Richards, 2004) .

However, the number and types of healthy food stores are not evenly distributed in the city, which may lead to disparities in accessibility of residents to healthy food stores. Studies have proved that low-income areas have a lower density of healthy food stores and higher food prices compared to higher income areas (Hendrickson et al., 2006).

The uneven distribution of healthy food stores in city may result in the lack of healthy food accessibility for some community and the formation of food deserts can be observed. Food desert are commonly defined as areas with restricted access to healthy and affordable foods as well as a variety of other nutritious options (USDA ERS, 2013), which is more likely to be observed neighborhoods where disadvantaged groups concentrate (Black et al., 2014). In order to reduce the food desert and promote better access to healthy food for the people of the city, the neighborhood food environment should be emphasized.

\section{2. methodologies of accessibility measurement}

Accessibility measures how easy it is from the origin to the specified destination (Widener \& Shannon, 2014). Previous literature often uses GIS to calculate the accessibility of healthy foods based on the location of residential areas. Some early studies measure the accessibility of residents to healthy foods by the service radius of food stores based on the Euclidean distance, but it ignores the impact of the actual environment on accessibility of healthy foods. Recent literatures often use a road network-based approach and begin to study the situation of people's actual healthy food accessibility in the city. However, they don't consider the differences associated with the use of different travel methods. Some scholars have found that private vehicles play a key role in improving access to health foods in western countries (Wang et al., 2014). The situation is different in China that residents 
often get health foods by walking or public transits. We should consider the impact of residents' transportation patterns in the actual food acquisition process. Meanwhile, food access is not only geographically restricted, but also subject to time constraints (Chen and Clark, 2013). Travel time is affected by traffic conditions, and we should calculate the travel time and the time spent on waiting (Farber, Morang, and Widener, 2014). Differences in time costs can also affect accessibility of healthy foods.

\section{Methodology and data}

\subsection{Research scale and method}

The 2018 edition of "Urban Residential Area Planning and Design Standards" stipulates that a 10-minute living circle should be equipped with a vegetable farm. Therefore, this paper takes the community level corresponding to the living circle as the research scale, and studies the community of Shanghai as a research object, with a total of 5432 communities.

Our method is comprised of 3 stages: travel time estimation, accessibility metrification, and spatial autocorrelation. In the first stage, we calculate travel time (e.g. bus and walking) from each community in Shanghai to its nearest health food store. In the second stage, these travel times are converted into measurements of accessibility and temporal variability. In the third stage, we calculate the community's average travel time and analyze the global and local spatial autocorrelation in Shanghai.

\subsection{Data}

The research data is divided into three parts: community, health food store and travel time. First, the data and location at the community level are from the sixth census in Shanghai. Second, the health food store mainly obtains its geographical location. The study is based on the map of Gaode. Platform, crawling Shanghai POI data in 2018, and screening various stores that provide healthy food, including vegetable shops, fruit shops, seafood seafood markets, supermarkets and farmer's markets, etc., screening a total of 18,526 data; third, travel time is mainly Based on the Gaode map platform, climb the real-time bus route planning time and collect the changes in travel time from each community to the health food store.

\section{Community-level healthy food reachable time}

\subsection{Status of Shanghai Health Food Store}




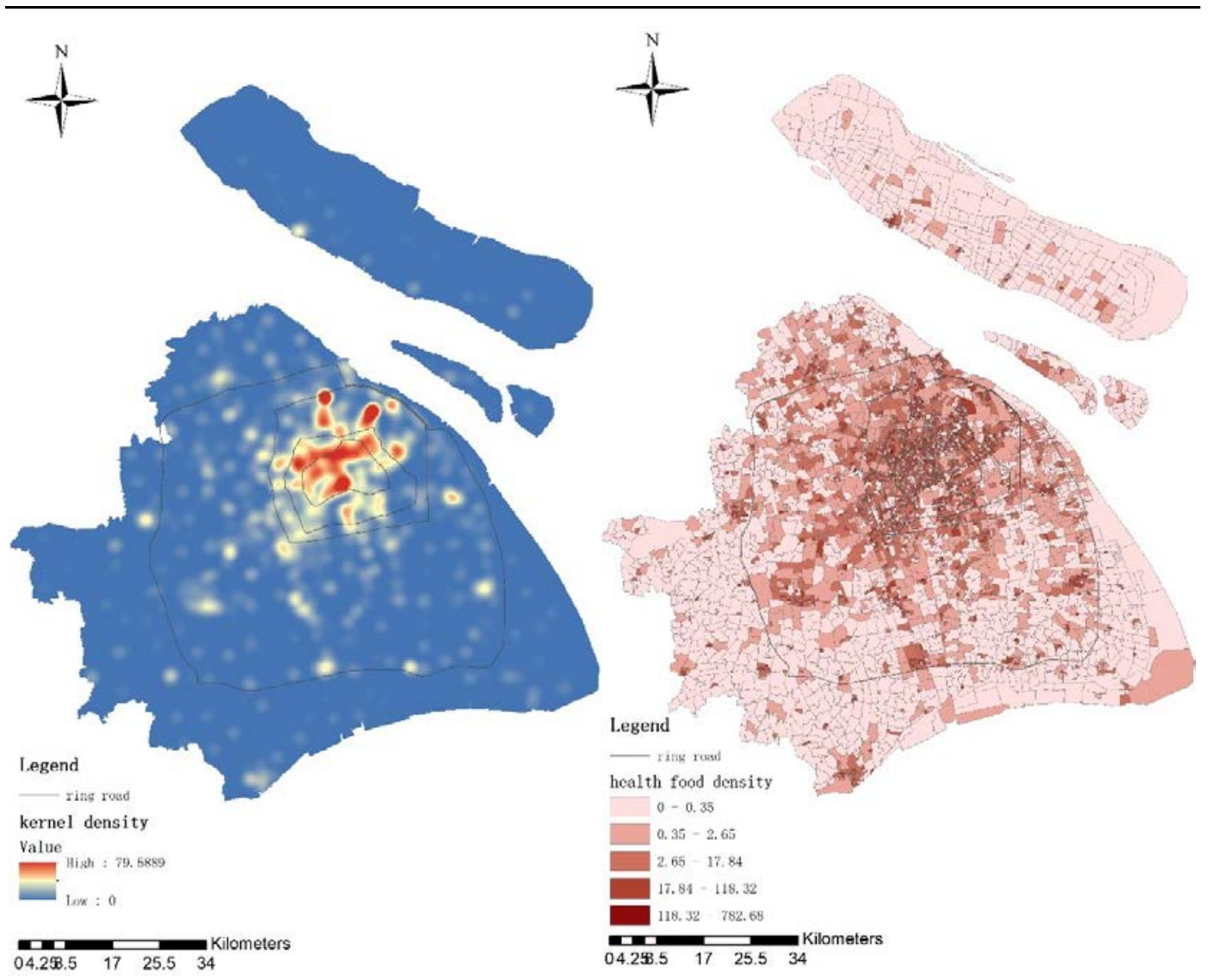

Figure 1 the kernel density of healthy food store

Figure 2 the density of healthy food store

There are a total of 18,526 health food stores in Shanghai. The concentration level of healthy food store in Puxi is significantly higher than that in Pudong. There are four loop lines in Shanghai and the density of healthy food store is highest in inner ring road. The northern part of the middle ring road to the inner ring road is relatively high concentration, and this area has radioactive extension to the outer ring area. There are fewer healthy food stores outside the outer ring line, which are distributed in spots. In the community level, there are no healthy food stores in 1879 communities, one healthy food store in 806 communities, and two health food stores in $\mathbf{5 8 9}$ communities. The Jixiang community in Hongkou District has the highest density of healthy food stores-782.0 per square kilometers.

\subsection{Measurement of travel time}

The Gaode Map (https://www.amap.com/) is China's leading electronic navigation platform. It uses the door-to-door method (Salonen \& Toivonen, 2013) to find the shortest travel route in the road network and based on real-time traffic information (including traffic volume, speed, sidewalk, one-way and restricted turns), It can provide a more realistic travel time calculation. The Gaode Map is employed to calculate the travel time of the community to the health food store.

The specific way is as follows: first, we use ArcGIS to calculate the geometric center of all communities in Shanghai. Based on the Shanghai road for network analysis, we use these community geometric centers as the origin, all healthy food stores in the city as the 
destination and calculate the travel time of a community to all health food stores. Later, we select the nearest travel time for a community and pair the community with the nearest healthy food store. Second, we develop a crawling tool to harvest the daily travel time from each community to each healthy food store during 6:00-22:00 every 20 minutes on the 14th June 2019. The crawling tool is written by Python from the web pages of Gaode Maps. The travel time collected includes the time of walking to bus station, travel time, and waiting time. The data of 5380 communities in Shanghai is collected, however, 52 communities cannot be located in network analysis, so the data is missing. Considering people's shopping habits, bus and health store business hours, the data collection period is determined. During the data collection process, when the community is close to the nearest healthy food store, the public transportation time will be converted into walking time, which is more in line with the real situation to measure the actual shopping behavior of the residents.

\subsection{Average travel time and changes in the community}

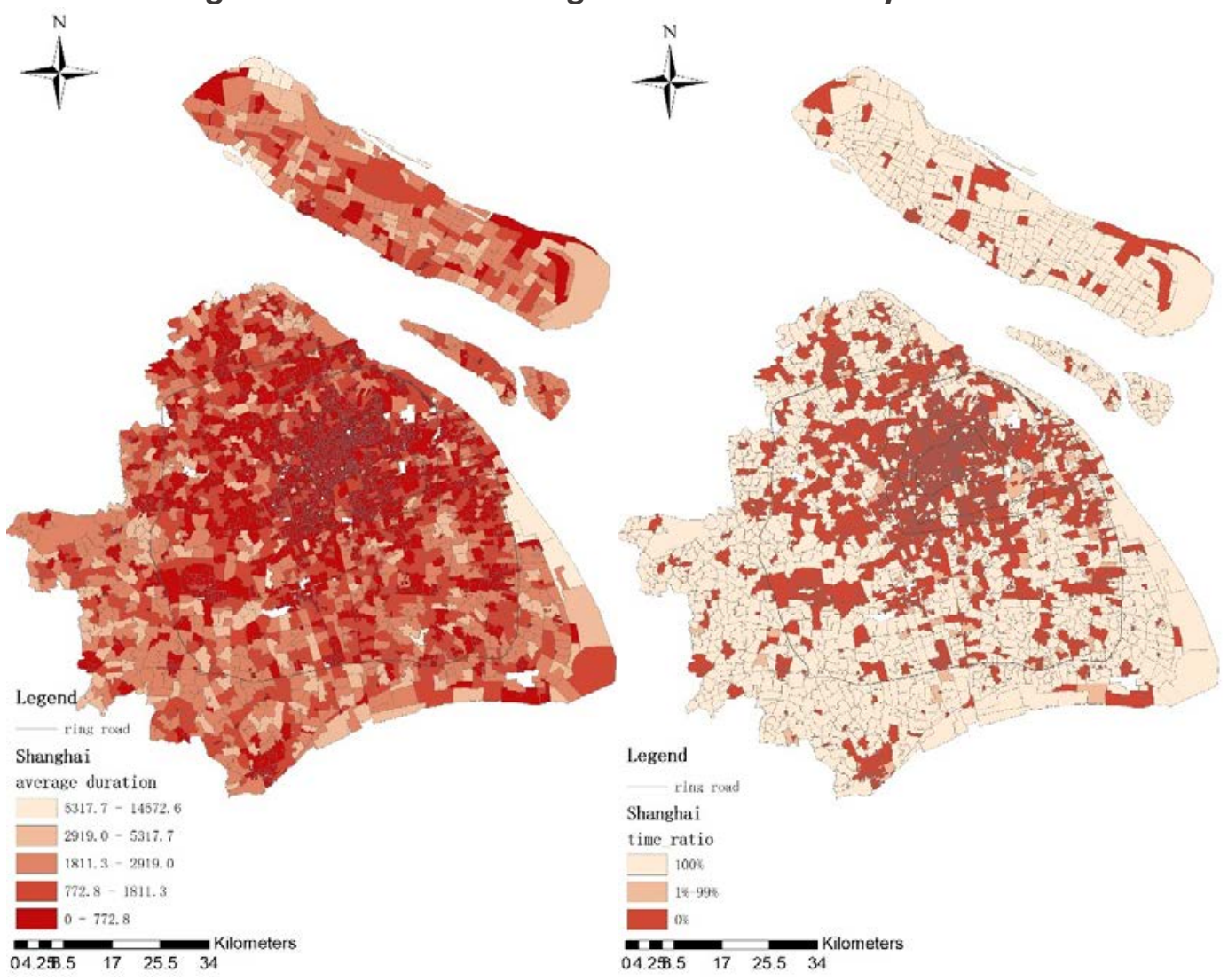

Figure 3 average travel time for healthy food in the community

Figure 4 the percentage of time that the community gets healthy food within 15 minutes

We calculate the travel time collected throughout the day to measure the accessibility of communities to healthy foods. The shorter average travel time is, the higher the accessibility of the community is. The results show that the average travel time of residents within middle ring road is shorter, and the accessibility to healthy foods is higher.

The accessibility of the south and east part of the area between the outer ring road and the middle ring road is good, and the accessibility of some communities in the north is slightly 
poor. From the suburban ring road to the outer ring road area, the travel time of the community has gradually increased, and the accessibility has gradually weakened. However, there are still some plaque-like communities with good accessibility of healthy food. The community outside the suburban ring road has a longer average travel time, and the accessibility of healthy food in these communities is poor, especially in the eastern coastal areas of Pudong District and the northern part of Chongming County.

Based on the 2018 edition of Urban Residential Area Planning and Design Standards, the concept of a fifteen-minute living circle plan is proved. We measure whether community residents can reach the nearest healthy food store within 15-minute travel and calculate the percentage of time that residents can reach the nearest health food store within 15 minutes during the day in order to measure the accessibility from a time perspective. The average city community in Shanghai has a healthy food travel time of 738.0 seconds, which basically meets the requirements of the 15-minute living circle for the vegetable market planning. Specifically, there are 3,885 communities in the city that can reach healthy food stores in 15 minutes, and the proportion of time that 114 communities reach healthy food stores within 15 minutes is between $0-1$. However, 1381 communities can't reach the health food store in 15 minutes all day, which affected by both long-distance and poor traffic conditions in this part of the region.

\subsection{Community case}

The travel time from the community to the healthy food store changes with the variation of traffic volume. We take the following six communities as examples to draw a travel time change chart for one day. It can be found that the travel time of the Gaoxiong Road community is short travel time and doesn't change. However, the travel time of other communities have some change during the day. Some communities have shown an increase in peak travel time in the morning and evening, which is consistent with the traffic pressure. 


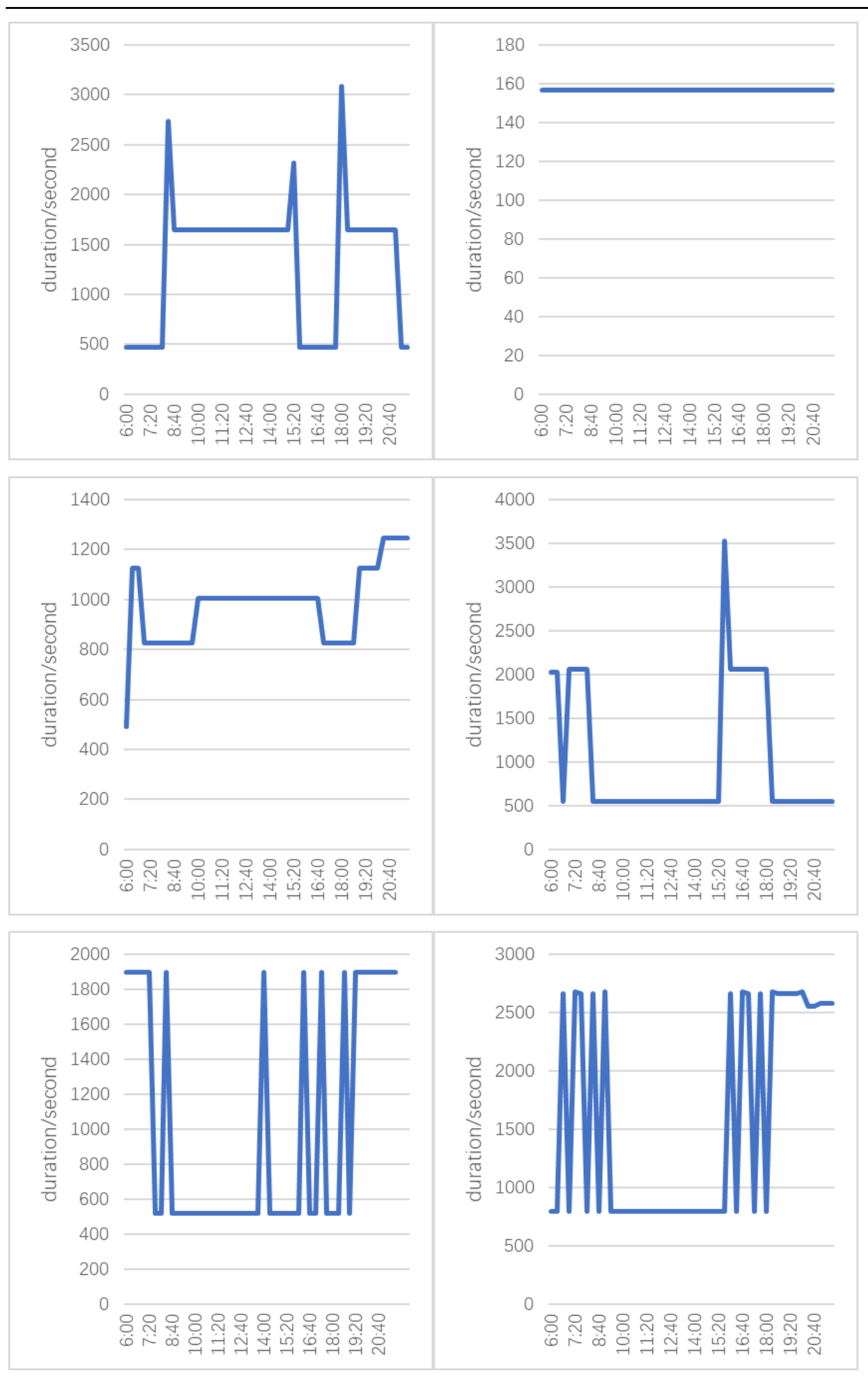

Figure 5 Travel time 1) Dongsenhanbi community, 2)Gaoxiong Road community, 3)Huakang community, 4) Xinmeigonghe community, 5) Shiwan community, 6)Gonghe villiage. 
As for the travel time during the day, the Gaoxiong Road community in Huangpu District is the shortest, and the proportion of time is $100 \%$ that healthy food store can be reached within 15 minutes of the day. The Dongshenhanbi community in Yangpu District and the Xinmei Gonghe Community in Jing'an District are both close to its nearest healthy food store, however, travel time is greatly affected by traffic conditions, and the proportion of time is also affected that healthy food store can be reached within 15 minutes of the day.

Table 1 Case community travel time

\begin{tabular}{|c|c|c|c|}
\hline community & district & Average travel time & $\begin{array}{l}\text { Proportion of time that healthy } \\
\text { food store can be reached } \\
\text { within } 15 \text { minutes of the day }\end{array}$ \\
\hline $\begin{array}{l}\text { Dongsenhanbi } \\
\text { community }\end{array}$ & $\begin{array}{l}\text { Yangpu } \\
\text { District }\end{array}$ & 1319.4 & $66.7 \%$ \\
\hline $\begin{array}{l}\text { Gaoxiong } \\
\text { community }\end{array}$ & $\begin{array}{l}\text { Huangpu } \\
\text { District }\end{array}$ & 156.8 & $100 \%$ \\
\hline Huakang community & Xuhui District & 979.2 & $66.7 \%$ \\
\hline $\begin{array}{l}\text { Xinmeigonghe } \\
\text { community }\end{array}$ & $\begin{array}{l}\text { Jing'an } \\
\text { District }\end{array}$ & 1020.5 & $29.2 \%$ \\
\hline Shiwan community & $\begin{array}{l}\text { Pudong } \\
\text { District }\end{array}$ & 1035 & $37.5 \%$ \\
\hline Gonghe villiage & $\begin{array}{l}\text { Minhang } \\
\text { District }\end{array}$ & 1563.3 & $41.7 \%$ \\
\hline
\end{tabular}

\section{Spatial autocorrelation}

\subsection{Method}

Spatial autocorrelation is a measure of the correlation of the same variable in different spatial locations. Since the spatial autocorrelation is attributed to the geographical location, the closer the spatial location is, the more similar it is (Lin Jinyao, Li Xia, 2014). Using the spatial weight matrix, the similarity of the position is measured. Based on the average travel time of healthy foods in the community, the spatial autocorrelation of healthy food accessibility in Shanghai community is studied. We use the GEODA software to construct the spatial weight matrix of the data through the Queen method. Spatial autocorrelation is further divided into global spatial autocorrelation and local spatial autocorrelation and we combine the two method to study.

1) Global spatial autocorrelation

The global spatial autocorrelation can measure the overall distribution of the elements and determine whether this phenomenon exists in the space with agglomeration characteristics. The Moran's I reflects the similarity of the spatial unit properties of spatially adjacent regions. 
The Moran's I is used as the global autocorrelation statistic, and the value range is $[-1,1]$. At a given level of significance, when the Moran's I is significantly positive, there is a significant positive correlation between the observations, and high (low) observations tend to cluster with high (low) observations, presenting a pattern of high agglomeration and low agglomeration. When the Moran's I is significantly negative, there is a significant negative correlation between the observations, and high observations tend to be clustered with low observations, presenting a spatially dispersed pattern (Xie Zhengfeng, Wang Qian, 2009). The Moran's I is as follows:

$$
I=\frac{n \sum_{i=1}^{n} \sum_{j=1}^{n} w_{i j}\left(x_{i}-\bar{x}\right)\left(x_{j}-\bar{x}\right)}{\sum_{i=1}^{n} \sum_{j=1}^{n} w_{i j} \sum_{i=1}^{n}\left(x_{i}-\bar{x}\right)^{2}}=\frac{\sum_{i=1}^{n} \sum_{j \neq i}^{n} w_{i j}\left(x_{i}-\bar{x}\right)\left(x_{j}-\bar{x}\right)}{S^{2} \sum_{i=1}^{n} \sum_{j \neq i}^{n} w_{i j}}
$$

among them:

$$
S^{2}=\frac{1}{n} \sum_{i}\left(x_{i}-\bar{x}\right)^{2} \quad \bar{x}=\frac{1}{n} \sum_{i=1}^{n} x_{i}
$$

It is verified by standardization statistic $Z$ whether there are spatial autocorrelation relations in $\mathrm{n}$ regions.

$$
Z=\frac{I-E(I)}{\sqrt{\operatorname{VAR}(I)}}
$$

2) Local spatial autocorrelation

Local spatial autocorrelation is a local indicator that measures the spatial connection of each unit. It is an indicator that describes the degree of spatial aggregation between regional units with significant similar values around the unit. Local metrics for spatial associations of all units are proportional to global spatial metrics. The Moran's I is as follows:

$$
I_{i}=\frac{\left(x_{i}-\bar{x}\right)}{S^{2}} \sum_{j} w_{i j}\left(x_{j}-\bar{x}\right)=\frac{n z_{i} \sum_{j} w_{i j} z_{j}}{z^{T} z}=z_{i}^{\prime} \sum_{j} w_{i j} z_{j}^{\prime}
$$

The local Moran's I is verified by the normalized statistic Z:

$$
Z\left(I_{i}\right)=\frac{I_{i}-E\left(I_{i}\right)}{\sqrt{\operatorname{VAR}\left(I_{i}\right)}}
$$




\subsection{Spatial autocorrelation results}

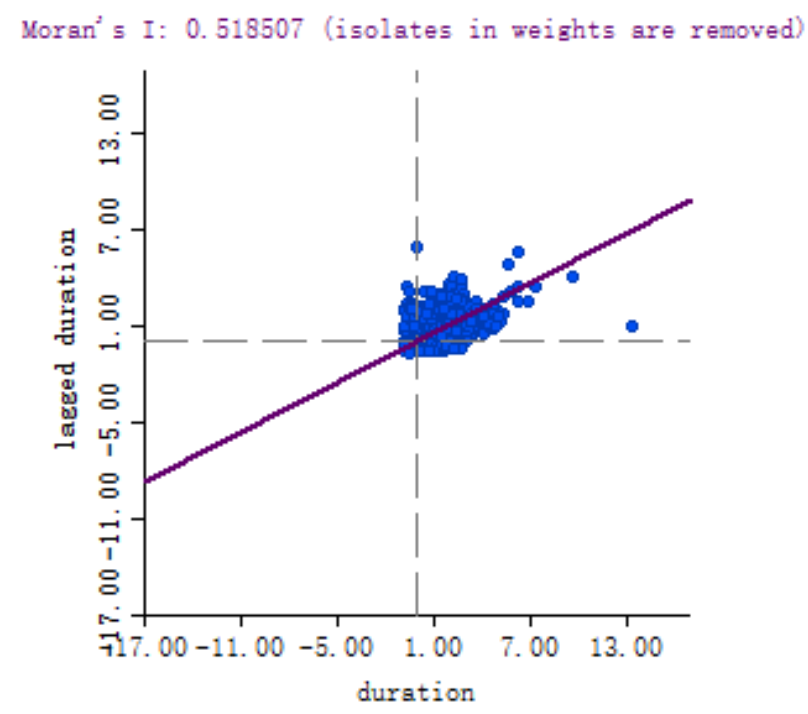

Figure 6 Moran's I of the average travel time of the community.

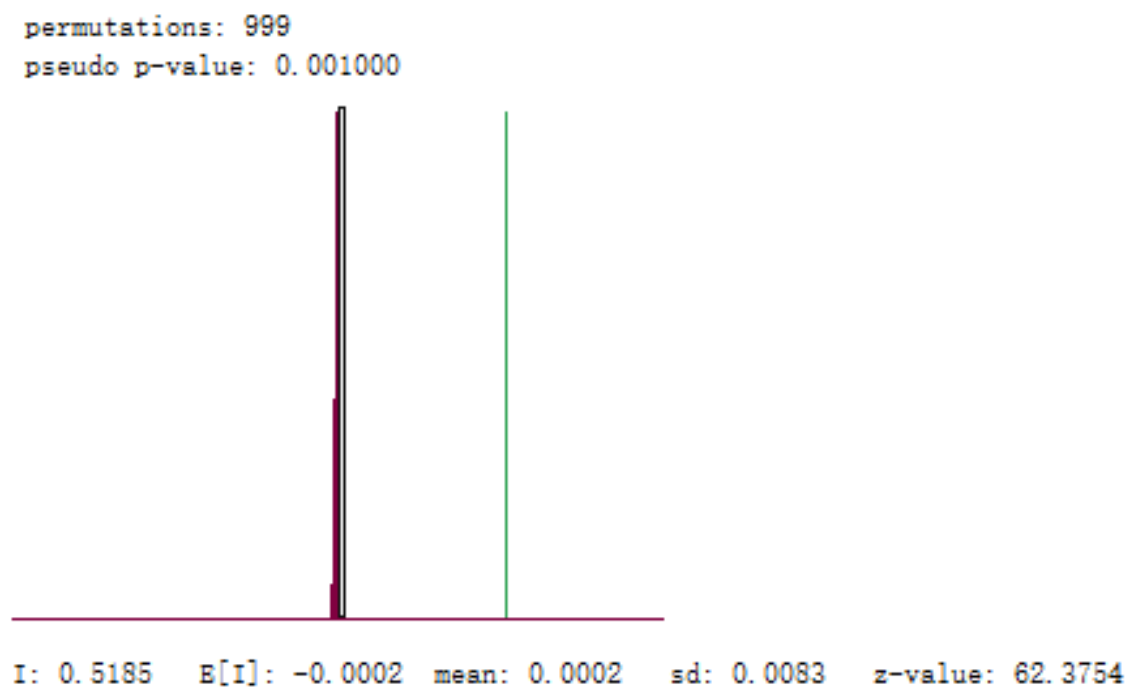

Figure 7 Random test of community average travel time

The Moran's I of the average travel time of the Shanghai community is 0.518507 , and the global indicator is 999 tests $p=0.001$, which is significant in the case of $99.9 \%$. The $Z$ value is 62.3754 , which is positive and significant, indicating that the average travel time of healthy foods in Shanghai community is not completely random in space, but shows spatial agglomeration and positive spatial autocorrelation with similar observations. (high or low values) tend to spatially aggregate. That is to say, in Shanghai, the areas where the community has a higher (or lower) average travel time for healthy foods tend to be adjacent to the communities with higher (or lower) average travel time. 


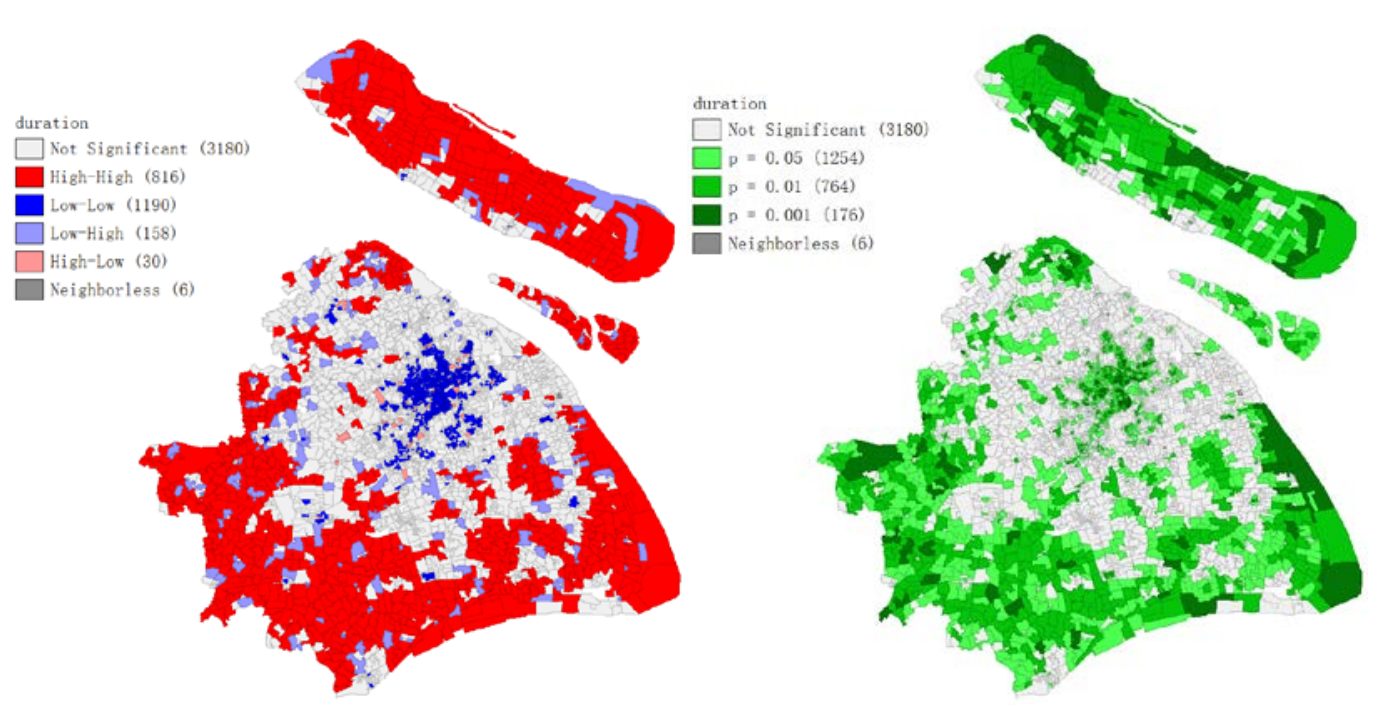

Figure 8 community average travel time local spatial autocorrelation and significance level

Most of the urban core areas within the Shanghai middle ring road are low-low areas. The average travel time of the communities themselves and surrounding communities is low. The spatial difference in this area is small, and there is strong spatial positive correlation. There are a few high-low areas around the core area. The average travel time of the community is higher but the surrounding level is lower and the spatial difference is larger. In fact, this may be due to the overall dispersal and local concentration of health food stores in the suburbs. Most of the areas outside the suburban ring road are high-high areas. The average travel time of the community and surrounding communities are high and the spatial difference is small. There is a strong spatial positive correlation. The level of significance is generally significant in the central and far-suburban areas of the city, and is not significant in the periphery of the city center.

The analysis found that the population density in the central area as well as the accessibility of healthy foods in the community were both high, and the population density in the peripheral areas as well as the accessibility of the community health foods were both low. There was a high correlation that the concentration of the population promotes the increase in the density of health food stores, and the facilities are closely related to the service population under market regulation. The distribution center of the health food store in Shanghai is still very prominent, showing a high center and a low level at the periphery. The centrality of the spatial distribution of health food stores in Shanghai is very prominent, showing a high center and a low level at the periphery, which is also related to the development history of Shanghai. The old city center of Shanghai, like Huangpu District and Jing'an District, has a long development period and has a long history of residents. All kinds of facilities are well-equipped and relatively livable, forming a strong centrality. With the development of Shanghai's urban construction, the central city has been expanding, making population and land development continue to spread to the periphery, and the transportation network is expanding outward. Under the regulation of the market economy, healthy food stores are also opening up with the accumulation of population. The expanding 
scope of population agglomeration has led to the formation of a transitional zone with low accessibility, low or non-aggregation areas between urban center and peripheral areas.

In general, the accessibility of community-level healthy foods in Shanghai has a layer structure. The central area has strong accessibility, while the accessibility of the peripheral areas is weak. Meanwhile, both areas have autocorrelation, and also show a certain centerperipheral structure.

\section{Conclusions and Discussion}

\subsection{Conclusions}

Healthy eating intake can improve people's health. If cities can better provide healthy food to residents, it can better promote healthy city development. This paper uses multi-source data to analyze the accessibility of healthy foods in Shanghai community residents. Firstly, based on the healthy food store POI, we use the ArcGIS software to analyze the basic situation of the city's healthy food stores, and find that there are a large number of healthy food stores in the core area of the city within the outer ring road. Meanwhile, the density of health food stores in the Puxi area is significantly higher than that in the Pudong area, and the healthy food stores outside the outer ring road are pointy agglomeration. Further, we collect the whole city travel time to the nearest health food store based on the Gaode map platform and get that the average time for the Shanghai community to get healthy food travel time is 738.0 seconds, which meets the requirements for the farm in the 15-minute living circle plan. The overall situation is good, but there are large differences between the regions. The communities within the outer ring road have higher accessibility to health food stores, and the accessibility of peripheral communities is lower. During the day, some of the communities are far away. Travel time is affected by traffic conditions, which causes certain differences in time costs.

Using the community average travel time of healthy foods for spatial autocorrelation analysis, it is found that average travel time is concentrated in the central and suburban areas, and the surrounding areas of the central area are scattered. The average travel time of healthy foods in the downtown area of Shanghai is low, that is, the accessibility of healthy foods is high. The accessibility of healthy foods in and around them is high, and the spatial difference is small. There is strong positive correlation. The accessibility of healthy foods in Shanghai suburbs is low for themselves and the surrounding areas and spatial differences are small. There is strong spatial correlation.

Meanwhile, the degree of accessibility between urban centers and peripheral areas is low. A transitional zone between the city center and the surrounding area that has a low degree of accessibility, high or low, or non-aggregation. This area is close to the central area and is the key area for future urban development and construction. The current conditions are obviously insufficient. It is urgent to take measures to improve the accessibility of healthy food in this area. In the long-term, efforts will be made to improve the accessibility of urban suburban communities, and gradually transform communities with insufficient accessibility into areas with better accessibility. 


\subsection{Discussion}

This paper mainly uses the POI data of healthy food stores. The POI data volume is large and it is easy to reflect the distribution of facilities. However, the POI data can only be expressed in the form of "points" and cannot reflect the service scope, grade, scale and other information of the facilities. It is difficult to conduct a more in-depth analysis. POI data has strong advantages in reflecting the spatial distribution and density of facilities, and can analyze the distribution structure or characteristics of the facilities. However, the in-depth study and discussion of facility accessibility should be further combined with urban population, transportation, land use and other aspects to analyze comprehensively.

This article discusses residents' access to healthy foods and only selects the accessibility of the nearest healthy food stores. It does not take into account other factors that affect residents' choice of healthy food stores, such as: price, healthy food type, consumption habits, etc. Future research should be combined with questionnaires, interviews and other research methods. From a human perspective, let us fully measure the availability of healthy foods.

\section{References}

C. Black, G. Moon, J. Baird (2014) Dietary inequalities: What is the evidence for the effect of the neighborhood food environment? Health \& Place, Vol.27.

D. Hendrickson, C. Smith, N. Eikenberry (2006) Fruit and vegetable access in four low-income food deserts communities in Minnesota, Agriculture and Human Values, Vol.23.

D. Rose, R. Richards (2004) Food store access and household fruit and vegetable use among participants in the US food stamp program, Public Health Nutrition, Vol.7.

E. Dowler (2001) Inequalities in diet and physical activity in Europe [J]. Public Health Nutrition, Vol.4 No.2B.

E.J. Taylor (2015) Fast food planning conflicts in Victoria 1969-2012: Is every unhappy family restaurant unhappy in its own way? Australian Planner, Vol. 52.

H. Wang, F. Qiu, B. Swallow (2014) Can community gardens and farmers' market relieve food desert problems? A study in Edmonton, Canada, Applied Geography, Vol.55J.

L. Kent, S. Thompson (2014) The three domains of urban planning for health and well-being, Journal of Planning Literature, Vol.29

Jamie Pearce, Rosemary Hiscock, Tony Blakely, Karen Witten (2009) A national study of the association between neighborhood access to fast-food outlets and the diet and weight of local residents, Health \& Place, Vol.15 No.1.

K. Morland, S. Wing, A. Diez Roux (2002) The contextual effect of the local food environment on residents' diets: the atherosclerosis risk in communities study, American Journal of Public Health, Vol, 92 No. 11.

M. Salonen, T. Toivonen (2013) Modelling travel time in urban networks: Comparable measures for private car and public transport, Journal of Transport Geography, Vol.31 
M. Story, K.M. Kaphingst, R. Robinson-O'Brien, K. Glanz (2008) Creating healthy food and eating environments: Policy and environmental approaches, Annual Review of Public Health, Vol. 29.

M.J. Widener, J. Shannon (2014) When are food deserts? Integrating time into research on food accessibility, Health \& Place, Vol.30.

S. Farber, M.Z. Morang, M.J. Widener (2014) Temporal variability in transit based accessibility to supermarkets, Applied Geography, Vol.53

S. Furey, C. Strugnell, H. Mcllveen (2001) An investigation of the potential existence of "Food Deserts" in rural and urban areas of Northern Ireland, Agriculture and Human Values, Vol.18

T.J. Key (2011) Fruit and vegetables and cancer risk, British Journal of Cancer, Vol.104

USDA ERS. Food access research atlas [online] (2013), http://www.ers.usda.gov/dataproducts/food-access-research-atlas/go-to-the-atlas.aspx

World Cancer Research Fund/American Institute for Cancer Research (2007) Food, Nutrition, Physical Activity, and the Prevention of Cancer: A Global Perspective, American Institute for Cancer Research, Washington, DC

World Health Organization (2013) Obesity and overweight, World Health Organization.

World Health Organization (2002) Report of the Joint WHO/FAO Expert Consultation on Diet, Nutrition and the Prevention of Chronic Diseases, WHO Technical Report Series 916. Geneva 